\title{
Extended excitons and compact heliumlike biexcitons in type-II quantum dots
}

\author{
Bhavtosh Bansal, ${ }^{1, *}$ S. Godefroo, ${ }^{1}$ M. Hayne, ${ }^{2, \dagger}$ G. Medeiros-Ribeiro, ${ }^{3, \ddagger}$ and V. V. Moshchalkov ${ }^{1, \S}$ \\ ${ }^{1}$ Institute for Nanoscale Physics and Chemistry (INPAC), Pulsed Fields Group, KU Leuven, Celestijnenlaan 200D, \\ Leuven B-3001, Belgium \\ ${ }^{2}$ Department of Physics, Lancaster University, Lancaster LA1 4YB, United Kingdom \\ ${ }^{3}$ Laboratório Nacional de Luz Síncrotron, P.O. Box 6192, 13084-971 Campinas, SP, Brazil \\ (Received 31 July 2009; revised manuscript received 4 October 2009; published 18 November 2009)
}

\begin{abstract}
We have used magnetophotoluminescence measurements to establish that InP/GaAs quantum dots have a type-II (staggered) band alignment. The average excitonic Bohr radius and the binding energy are estimated to be $15 \mathrm{~nm}$ and $1.5 \mathrm{meV}$, respectively. When compared to bulk InP, the excitonic binding is weaker due to the repulsive (type-II) potential at the heterointerface. The measurements are extended to over almost 6 orders of magnitude of laser excitation powers and to magnetic fields of up to $50 \mathrm{~T}$. It is shown that the excitation power can be used to tune the average hole occupancy of the quantum dots and hence the strength of the electron-hole binding. The diamagnetic shift coefficient is observed to drastically reduce as the quantum dot ensemble makes a gradual transition from a regime where the emission is from (hydrogenlike) two-particle excitonic states to a regime where emission from (heliumlike) four-particle biexcitonic states also becomes significant.
\end{abstract}

DOI: $10.1103 /$ PhysRevB.80.205317

PACS number(s): 78.67.Hc, 71.35.Ji

\section{INTRODUCTION}

Until recently-but for a few exceptions - the study of quantum-dot (QD) heterostructures with the staggered (typeII) band alignment had been largely ignored because of the absence of confinement of one of the two types of carriers and their presumed poor radiative efficiency. However, it has come to be recognized that these structures are interesting, especially for their rich physics of excitons. ${ }^{1-7}$

In contrast to the usual type-I QDs (e.g., InAs/GaAs), where the confinement energy scale is far greater than the energy of the Coulomb interaction, the role of confinement in type-II QDs is largely limited to defining the geometry of the system. This in itself has interesting consequences. The multiply connected topology can give rise to an oscillatory ground-state energy for the magnetoexcitons.,2 Second, type-II QDs also act as nanocapacitors ${ }^{8}$ which selectively accommodate only one type of particles; but once charged they can bind the complementary particle to form an exciton. The strength of the Coulomb interaction can be modified by screening or magnetic field. ${ }^{4}$ At higher excitation powers, they can be doubly charged and form four-particle bound states (biexcitons). The biexcitons in a type-II QD system are very unlike their counterparts ${ }^{9}$ in type-I QDs and quantum wells. They always have negative "binding energy." In the atomic physics language, while the usual biexciton is structurally analogous to a hydrogen molecule, the biexciton ${ }^{6}$ in type-II QDs is more like a helium atom. ${ }^{5}$

In this paper we have probed the nature of the ensemble photoluminescence (PL) emission from a sample with InP QDs in a GaAs matrix. Although the band offsets ${ }^{10}$ of bulk InP and GaAs and some previous studies suggest that this material combination forms type-II structures with electrons localized within the InP quantum dots and free holes in the GaAs matrix, the energy gap of InP and GaAs is within 100 $\mathrm{meV}$ of each other, and the conduction-band offset is relatively small. ${ }^{10}$ Thus alloying and anisotropic strain within the
QDs ${ }^{11}$ can modify the energy gap and the relative offsets in a way that is dependent on the details of the size and shape of the QDs. For example, a comprehensive $k \cdot p$ calculation $^{10}$ does not find a type-II alignment in this system. Second, thick $(>100 \mathrm{~nm})$ InP heteroepitaxial layers on GaAs and even homoepitaxial InP have shown a broad emission peak at $1.34 \mathrm{eV}$ (Ref. 12) due to donor-acceptor (DA) pair recombination, which is rather close to the PL energy of InP/GaAs QDs. Furthermore, the DA emission has characteristics ${ }^{13}$ generally used to classify spatially indirect excitons from type-II QDs-excitation-power-dependent blueshift, ${ }^{3,14}$ spectrally broad emission at a subbandgap energy in macro-PL, and narrow emission lines from localized states in micro-PL. ${ }^{3}$ One of the aims of this study is to unambiguously establish the type-II band alignment in this system and highlight the role of heterostructure boundary conditions on the size and the binding energy of excitons. In this work we will not discuss the Aharonov-Bohm-type effects associated with the topology of the wave function as these have already been extensively discussed in literature ${ }^{1,2}$ and manifest on energy scales an order of magnitude smaller than we are interested in here.

Second, by extending the measurements over 6 orders of magnitude of excitation power, we have been able to change the average electronic occupancy within the QDs. This leads to significant changes in the diamagnetic shift coefficient that are a result of the interesting physics of biexcitons.

\section{EXPERIMENT}

The sample was grown by metal-organic vapor-phase epitaxy on a (001) GaAs substrate. ${ }^{11}$ The QD density was about $3 \times 10^{10} \mathrm{~cm}^{-2}$ with an average diameter of $32 \mathrm{~nm}( \pm 6 \mathrm{~nm})$ and average height of about $4 \mathrm{~nm}( \pm 2 \mathrm{~nm})$ as measured by cross-sectional transmission electron microscopy. ${ }^{11}$ But based on experience from other heterostructures, it is possible that the actual size of quantum dots is much smaller. ${ }^{15}$ 


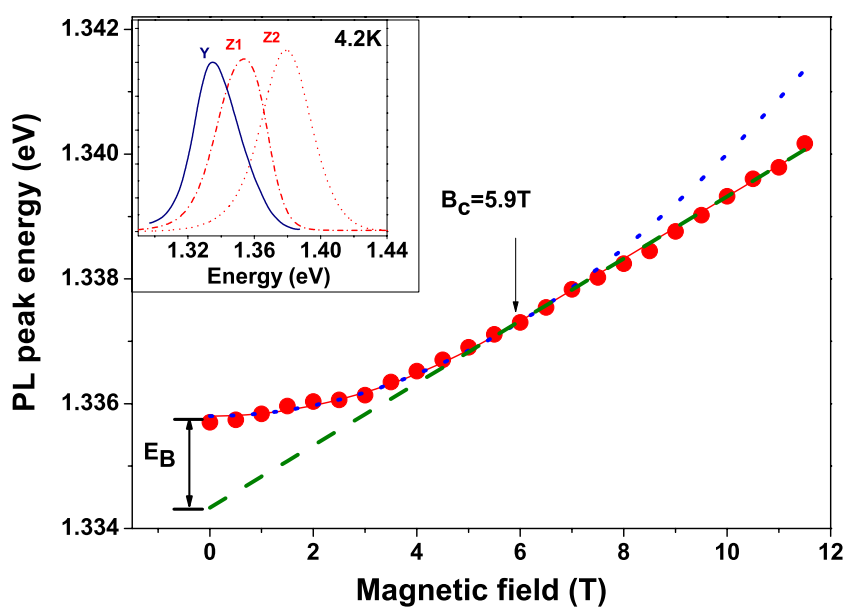

FIG. 1. (Color online) (Inset) Three representative spectra from the InP/GaAs QD ensemble taken under different conditions. Y:magnetic field $B=0$, laser power $P \sim 10^{-4} \mathrm{~W} \mathrm{~cm}^{-2}, \mathrm{Z} 1: B=0$, $P \sim 85 \mathrm{~W} \mathrm{~cm}^{-2}, \mathrm{Z} 2: B=50 \mathrm{~T}, P \sim 85 \mathrm{~W} \mathrm{~cm}^{-2}$. The relative amplitudes have been arbitrarily scaled. (main figure) The measured peak shift as a function of magnetic field at the excitation power of about $10^{-4} \mathrm{~W} \mathrm{~cm}^{-2}$. (solid line) Fit to Eq. (1) (dotted lines) Eqs. (1a) and (1b) plotted separately. The crossover from Coulombic confinement to magnetic confinement occurs at $5.9 \mathrm{~T}$ and corresponds to an effective Bohr radius of $15 \mathrm{~nm}$. The extrapolation of the linear slope measured at high field to zero field gives the binding energy, $E_{B}=1.5 \mathrm{meV}$.

Nonresonant (excitation wavelength=532 nm) PL measurements were performed at liquid-helium temperature with the excitation power varied between $\sim 10^{-4}-100 \mathrm{~W} \mathrm{~cm}{ }^{-2}$. The light from the excitation laser and the sample PL was fiber-optically coupled in and out of (i) a variable-temperature cryostat within the bore of a superconducting magnet $(B \leq 12 \mathrm{~T})$ for magneto-PL measurements at low excitation powers and (ii) a liquid-helium bath cryostat whose tail was within the $18 \mathrm{~mm}$ bore of a pulsed field coil $(B \leq 50 \mathrm{~T})$ for measurements at higher powers $P>30 \mathrm{~W} \mathrm{~cm}^{-2}$. The PL spectra were recorded by an electron-multiplying charged-coupled device after being dispersed by a $30 \mathrm{~cm}$ imaging spectrograph. Pulsed magnetic fields of up to $50 \mathrm{~T}$ were generated using a $5 \mathrm{kV}, 500 \mathrm{~kJ}$ capacitor bank. The field had a duration of about $20 \mathrm{~ms}$, during which several PL spectra were recorded. Figure 1 (inset) shows three representative spectra under different conditions.

\section{RESULTS AND DISCUSSION}

\section{A. Excitons: Type-II band alignment}

Figure 1 shows the magnetic field-dependent PL peakshift measured at $4.2 \mathrm{~K}$ at a very low excitation power of about $10^{-4} \mathrm{~W} \mathrm{~cm}^{-2}$. Using the model of a hydrogenic exciton, one can semiphenomenologically describe this shift by the following equations, ${ }^{4,5}$

$$
\begin{gathered}
E(B)=E_{0}+\frac{q^{2}\left\langle\rho_{x}^{2}\right\rangle}{8 \mu} B^{2}, \quad \text { for } B<B_{c}, \\
E(B)=E_{0}-\frac{\hbar^{2}}{2 \mu\left\langle\rho_{x}^{2}\right\rangle}+\frac{\hbar q}{2 \mu} B, \text { for } B>B_{c} .
\end{gathered}
$$

These equations are derived under the assumption that the magnetic field-induced change in the ground-state energy of a hydrogenic exciton from the low-field regime of quadratic (diamagnetic) shift to the high-field linear shift (due to transitions between effectively free Landau levels) is adiabatically continuous (i.e., its functional form is continuous and differentiable) between two well-defined limits. Note that the high-field limit is approximate as it assumes that the transitions are between free Landau levels and ignores the weak $\left(\log ^{2} B\right.$, as $\left.B \rightarrow \infty\right)$ dependence $^{16}$ of the excitonic binding energy on magnetic field. Here $E_{0}$ is the ground-state energy without the magnetic field, $B_{c}=2 \hbar /\left(q\left\langle\rho_{x}^{2}\right\rangle\right)$ corresponds to the magnetic field when $2 l_{B}^{2}=\left\langle\rho_{x}^{2}\right\rangle, \rho_{x}$ is the excitonic Bohr radius, $l_{B}$ is the magnetic length, $\mu$ is the reduced effective mass, and $q$ the magnitude of the electronic charge. The fit to Eq. (1), along with its physical content, is also shown in Fig. 1. The second term in Eq. (1b) corresponds to the excitonic binding energy and is the extrapolation of the high-field slope [third term in Eq. (1b)] to $B=0 .{ }^{17}$ The crossover from quadratic to linear slope is found at $5.9 \mathrm{~T}$. The values of the exciton radius, the binding energy, and the diamagnetic shift coefficient are given in Table I.

Table I also compares the effect of the heterostructure boundary conditions on the excitonic parameters in InP. Note the systematic trend in the value of the diamagnetic shift coefficient. The effective electron-hole interaction in type-II

TABLE I. Comparison of exciton parameters in InP under different boundary conditions.

\begin{tabular}{lccc}
\hline \hline & $\begin{array}{c}\text { Diamag. shift } \Gamma \\
(\mu \mathrm{eV} \mathrm{T})^{-2}\end{array}$ & $\begin{array}{c}\text { Bohr radius } \sqrt{\left\langle\rho^{2}\right\rangle} \\
(\mathrm{nm})\end{array}$ & $\begin{array}{c}\text { Binding energy } E_{B} \\
(\mathrm{meV})\end{array}$ \\
\hline InP/GaAs QD & $42.4 \pm 0.5$ & $15.0 \pm 0.1$ & $1.5 \pm 0.1$ \\
Bulk InP & $40^{\mathrm{c}}$ & $\sim 12^{\mathrm{b}}$ & $\begin{array}{c}.8 \pm 0.2^{\mathrm{d}} \\
\text { InP/GaInP QD }\end{array}$ \\
\hline \hline
\end{tabular}

aThis work.

${ }^{b}$ Estimated from the diamagnetic shift in the first column using electron mass of InP, $\mu=0.08 m_{0}$.

${ }^{\mathrm{c}}$ Reference 19.

${ }^{\mathrm{d}}$ Reference 20.

${ }^{\mathrm{e}}$ References 18 and 21 . 


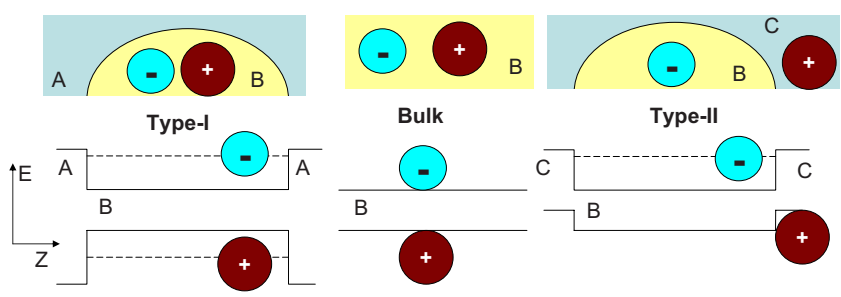

FIG. 2. (Color online) Schematic depiction of an exciton in bulk material and heterostructured QDs with type-I and type-II band alignment. Material combination "B/A" has type-I alignment (e.g., InAs/InP and possibly InP/InGaP) whereas "B/C" has type-II band alignment (e.g., InP/GaAs). Type-I confinement results in a decrease in the exciton radius and an increase in the exciton binding energy. For QDs with type-II band alignment, the exciton binding should be weaker and the radius larger compared to the bulk InP values and the emission energy can be smaller than the band gaps of either of the two materials.

dots is the weakest followed by bulk InP, and then finally $\mathrm{InP} / \mathrm{Ga}_{x} \mathrm{In}_{1-x} \mathrm{P}$ QDs which show a much stronger binding (also see Fig. 2). Thus, on physical grounds, the observations are most consistent with type-II band alignment.

Next, we will explore the multiparticle states in these QDs by excitation-power-dependent PL measurements. These measurements will also help us rule out DA recombination, as will be discussed in Sec. III C. In what follows, we will assume that the QDs have type-II alignment.

\section{B. Biexcitons}

The PL peak position, measured without the magnetic field [Fig. 3(b)] is strongly excitation-power-dependent beyond an incident laser intensity of about $10^{-1} \mathrm{~W} \mathrm{~cm}^{-2}$. This marks the point where multiparticle states start to play a role in PL. The observed blueshift is due to the additional energy associated with the capacitive charging of the QDs. The integrated PL intensity (not shown here for brevity) also gradually changes its slope from linear to (slightly) superlinear beyond $10^{-1} \mathrm{~W} \mathrm{~cm}^{-2}$, indicating that a fraction of emission is from biexcitonic states.

The dependence of the PL peak position as a function of the magnetic field at different excitation powers, measured over almost 6 orders of magnitude, is shown in Fig. 3(a). Notice that the curves are all qualitatively similar and that they all can be fitted to Eqs. (1) [solid lines in Fig. 3(a)]. This is because the $B$-dependent change in the energy of biexcitonic levels is just the sum of single-particle energies, but with the important difference that the relevant radius is now the biexcitonic radius $\rho_{x x}$ and hence the diamagnetic shift coefficients are significantly different.

However, for an ensemble, the analysis is complicated by the facts that (i) the emission is from a mixture of excitonic and biexcitonic states with an unknown biexciton fraction $\alpha$, (ii) when a photon is emitted by a biexciton, what is measured in the magneto-PL experiment is the difference in the shifts of biexcitonic and excitonic levels, because the emission process involves biexciton $\rightarrow$ exciton + photon. The measured change in the PL emission energy at low magnetic fields [diamagnetic shift regime, Eq. (1a)] will then be
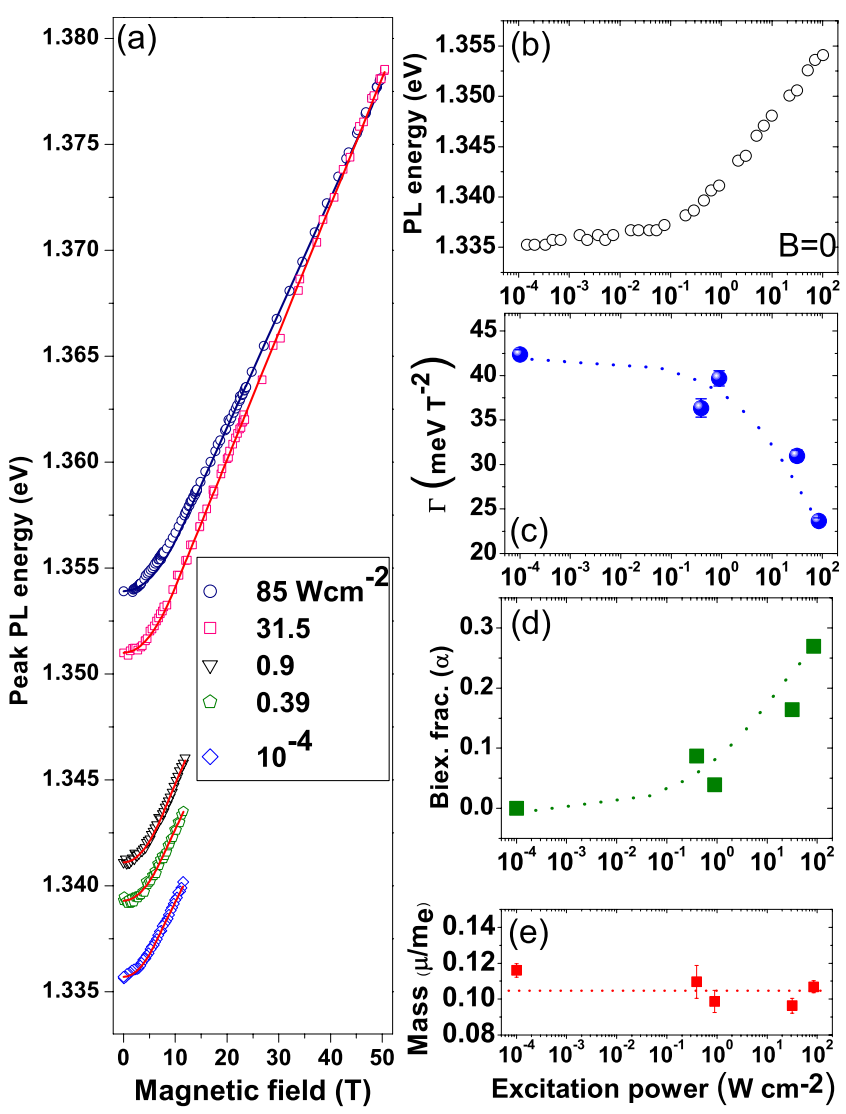

FIG. 3. (Color online) (a) The magnetic field-dependent shift of the emission peak at different excitation powers-0.0001, 0.39, 0.9, 32 , and $85 \mathrm{~W} \mathrm{~cm}^{-2}$. Pulsed field measurements, which allow for only a short photon accumulation time, could only be performed at high powers. (b) Dependence of the PL energy on the excitation power measured at $4.2 \mathrm{~K}(B=0)$. (c) The diamagnetic shift coefficient $(\Gamma)$. (d) The biexciton fraction roughly inferred from (c) using Eq. (2). $\rho_{x x} / \rho_{x}$ was assumed to be 0.6 , which is close to the ratio of hydrogen to helium radii. (e) The exciton reduced mass. The dotted lines in (c), (d), and (e) are just to guide the eye.

$$
\Delta E_{\mathrm{dia}}(B)=\frac{q^{2}}{8 \mu}\left[\alpha\left(2\left\langle\rho_{x x}^{2}\right\rangle-\left\langle\rho_{x}^{2}\right\rangle\right)+(1-\alpha)\left\langle\rho_{x}^{2}\right\rangle\right] B^{2} .
$$

The diamagnetic shift coefficient $\Gamma$ is thus expected to be strongly excitation-power dependent. Indeed this is observed in Fig. 3(c). $\Gamma$ changes by factor of 2 due to the much smaller biexcitonic radius. Recall that in type-I QDs, confinement usually renders the diamagnetic shift independent of the charge in the dot. ${ }^{9}$ In general, it depends on the relative extents of the spatial spread of the electron and the hole wave functions.

For a rough estimate of the biexciton fraction as a function of excitation power, we use the ratio of the Bohr radii for the helium and the hydrogen atom, $\rho_{\mathrm{He}} / \rho_{\mathrm{H}} \approx 0.6 .{ }^{22}$ Figure $3(\mathrm{~d})$ is plotted using this value of $\rho_{x x} / \rho_{x}$ and we find that the biexciton fraction at the highest excitation power is about $30 \%$. Also note that when $\left\langle\rho_{x x}^{2}\right\rangle /\left\langle\rho_{x}^{2}\right\rangle<0.5$, Eq. (2) predicts that the observed diamagnetic shift coefficient would not only reduce but also become negative at very high excitation powers. 
The strong dependence of the diamagnetic shift coefficient on the excitation power also explains the anomalous results of Godoy et al., ${ }^{23}$ who found the excitonic diameter to be smaller in InP/GaAs compared to the bulk InP. They measured a diamagnetic shift coefficient of between $5-20 \mu \mathrm{eV} \mathrm{T}^{-2}$, the latter of which is equal to what we measure at the highest excitation power.

Figure 3(e) shows that the exciton mass [high-field slope of the curves in Fig. 3(a)] stays at approximately $0.1 m_{e}$, constant within $15 \%$ over the whole range of excitation powers. Hence the changes in the diamagnetic shift coefficient can be understood as being largely the difference in the excitonic and biexcitonic radii [Eq. (2)]. This provides consistency to the analysis. The value of the mass is between the freeelectron mass in $\operatorname{InP}\left(0.08 m_{e}\right)$ and the free heavy hole in GaAs $\left(0.45 m_{e}\right)$. This is reasonable because the electron is largely immobilized by the quantum dot. Strain and nonparabolicity effects may further contribute to the enhancement of the electron mass.

\section{Donor-acceptor pair recombination hypothesis}

The excitation-power dependence of the diamagnetic shift coefficient [Fig. 3(c)] also rules out recombination due to overlap between donor and acceptor wave functions. DA recombination has an energy ${ }^{24}$

$$
\hbar \omega_{\mathrm{DA}}=E_{g}-E_{D^{0}}^{b}-E_{A^{0}}^{b}+\frac{e^{2}}{4 \pi \epsilon_{0} \epsilon r_{\mathrm{DA}}}-m \hbar \omega_{L O} .
$$

$E_{g}$ is the energy gap, $E_{D^{0}}^{b}$ and $E_{A^{0}}^{b}$ are the binding energies of the donor and acceptor levels, the fourth term is the Coulomb repulsion energy of the ionized centers after recombination, and $m \hbar \omega_{L O}$ are the phonon-assisted transitions. ${ }^{24}$ While the DA-pair emission shares some characteristics with emission from type-II QDs such as the diamagnetic shifts from DA recombination would be of the same order as for excitons (Fig. 1) and at higher excitation powers there would be a blueshift ${ }^{24}$ qualitatively similar to that seen in Fig. 3(b), the diamagnetic shift coefficient itself should not have an excitation-power dependence as observed in Fig. 3(c). Furthermore, one should expect a strong quenching of the PL intensity in magnetic field for DA recombination-the wave functions shrink in the magnetic field and since the electron and hole centers are not at the same point in space, their (exponentially small) overlap will be strongly reduced. This is also not observed.

\section{CONCLUSIONS}

We studied the PL from InP/GaAs QD heterostructures in very high magnetic fields $(B \leq 50 \mathrm{~T})$ over almost 6 orders of magnitude of excitation power. Magneto-PL measurements at very low excitation powers established that the excitons have an average Bohr radius of $15 \mathrm{~nm}$ and a binding energy of $1.5 \mathrm{meV}$. These values indicate a much weaker binding for the excitons in comparison with bulk InP and provide strong evidence for type-II band alignment in these QDs. We also studied the evolution of the electron-hole binding as the QD ensemble makes a gradual transition from a regime where the emission is from (hydrogenlike) two-particle excitonic states, to a regime where the emission from (heliumlike) four-particle biexcitonic states also becomes significant. This was demonstrated by a strong variation in the diamagnetic shift with the excitation power.

\section{ACKNOWLEDGMENTS}

The work at the KU Leuven is supported by the FWO and GOA programmes and by the Methusalem Funding of the Flemish Government. M.H. acknowledges the support of the Research Councils U.K.

\footnotetext{
*bhavtosh.bansal@gmail.com

†m.hayne@lancaster.ac.uk

¥gmedeiros@lnls.br

§victor.moshchalkov@fys.kuleuven.be

${ }^{1}$ E. Ribeiro, A. O. Govorov, W. Carvalho, and G. MedeirosRibeiro, Phys. Rev. Lett. 92, 126402 (2004); I. R. Sellers, V. R. Whiteside, I. L. Kuskovsky, A. O. Govorov, and B. D. McCombe, ibid. 100, 136405 (2008); A. B. Kalameĭtsev, V. M. Kovalev, and A. O. Govorov, JETP Lett. 68, 669 (1998).

${ }^{2}$ M. H. Degani, M. Z. Maialle, G. Medeiros-Ribeiro, and E. Ribeiro, Phys. Rev. B 78, 075322 (2008).

${ }^{3}$ M. K. K. Nakaema, F. Iikawa, M. J. S. P. Brasil, E. Ribeiro, G. Medeiros-Ribeiro, W. Carvalho, Jr., M. Z. Maialle, and M. H. Degani, Appl. Phys. Lett. 81, 2743 (2002).

${ }^{4}$ B. Bansal, M. Hayne, M. Geller, D. Bimberg and V. V. Moshchalkov, Phys. Rev. B 77, 241304(R) (2008).

${ }^{5}$ M. Hayne, J. Maes, S. Bersier, V. V. Moshchalkov, A. Schliwa, L. Müller-Kirsch, C. Kapteyn, R. Heitz, and D. Bimberg, Appl. Phys. Lett. 82, 4355 (2003); M. Hayne, J. Maes, S. Bersier, A.
}

Schliwa, L. Müller-Kirsch, C. Kapteyn, R. Heitz, D. Bimberg, and V. V. Moshchalkov, Physica B (Amsterdam) 346-347, 421 (2004).

${ }^{6}$ K. Matsuda, S. V. Nair, H. E. Ruda, Y. Sugimoto, and T. Saiki, Appl. Phys. Lett. 90, 013101 (2007).

${ }^{7}$ J. R. Madureira, Márcio P. F. de Godoy, Maria J. S. P. Brasil, and F. Iikawa, Appl. Phys. Lett. 90, 212105 (2007).

${ }^{8}$ M. Geller, C. Kapteyn, L. Müller-Kirsch, R. Heitz, and D. Bimberg, Appl. Phys. Lett. 82, 2706 (2003).

${ }^{9}$ N. I. Cade, H. Gotoh, H. Kamada, H. Nakano, and H. Okamoto, Phys. Rev. B 73, 115322 (2006).

${ }^{10}$ C. E. Pryor and M.-E. Pistol, Phys. Rev. B 72, 205311 (2005).

${ }^{11}$ R. L. Maltez, E. Ribeiro, W. Carvalho, Jr., D. Ugarte, and G. Medeiros-Ribeiro, J. Appl. Phys. 94, 3051 (2003).

${ }^{12}$ D. J. Olego, Y. Okuno, T. Kawano, and M. Tamura, J. Appl. Phys. 71, 4502 (1992); M. B. Derbali, J. Meddeb, H. Mâaref, D. Buttard, P. Abraham, and Y. Monteil, ibid. 84, 503 (1998).

${ }^{13}$ M. B. Derbali, J. Meddebb, and P. Abraham, Thin Solid Films 364, 192 (2000). 
${ }^{14}$ B. Wang and S.-J. Chua, Appl. Phys. Lett. 78, 628 (2001).

${ }^{15}$ R. Timm, H. Eisele, A. Lenz, S. K. Becker, J. Grabowski, T.-Y. Kim, L. Müller-Kirsch, K. Pötschke, U. W. Pohl, D. Bimberg, and M. Dähne, Appl. Phys. Lett. 85, 5890 (2004).

${ }^{16}$ R. P. Seisyan and B. P. Zakharchenya, Landau Level Spectroscopy, Modern Problems in Condensed Matter Sciences Vol. 27.1 (North-Holland, Amsterdam, 1991), pp 344-443.

${ }^{17}$ H. Weman, M. Potemski, M. E. Lazzouni, M. S. Miller, and J. L. Merz, Phys. Rev. B 53, 6959 (1996).

${ }^{18}$ M. Hayne, R. Provoost, M. K. Zundel, Y. M. Manz, K. Eberl, and V. V. Moshchalkov, Phys. Rev. B 62, 10324 (2000).

${ }^{19}$ M. P. F. de Godoy, M. K. K. Nakaema, F. Iikawa, W. Carvalho, Jr., E. Ribeiro, and A. L. Gobbi, Rev. Sci. Instrum. 75, 1947 (2004).
${ }^{20}$ S. B. Nam, D. C. Reynolds, C. W. Litton, T. C. Collins, P. J. Dean, and R. C. Clarke, Phys. Rev. B 13, 1643 (1976).

${ }^{21}$ M. Sugisaki, H.-W. Ren, S. V. Nair, K. Nishi, and Y. Masumoto, Phys. Rev. B 66, 235309 (2002).

${ }^{22}$ A proper theoretical calculation would require solving the Schrodinger equation for the four-body problem in presence of suitable boundary conditions and magnetic field, which is beyond the scope of this work.

${ }^{23}$ M. P. F. de Godoy, P. F. Gomes, M. K. K. Nakaema, F. Iikawa, M. J. S. P. Brasil, R. A. Caetano, J. R. Madureira, J. R. R. Bortoleto, M. A. Cotta, E. Ribeiro, G. E. Marques, and A. C. R. Bittencourt, Phys. Rev. B 73, 033309 (2006).

${ }^{24}$ C. Klingshirn, Semiconductor Optics, 2nd ed. (Springer, Berlin, 2005), p. 353. 